Bull. Austral. Math. Soc.

VOL. $71(2005) \quad[1-9]$

\title{
STRICTLY SINGULAR AND STRICTLY COSINGULAR LINEAR RELATIONS AND THEIR CONJUGATES
}

\author{
Teresa Álvarez
}

In this paper various conditions are given under which the strict singularity (respectively, strict cosingularity) of a linear relation implies the strict singularity (respectively, strict cosingularity) of its conjugate.

\section{INTRODUCTION}

In $([8,16,17])$, the authors have considered the notions of subprojective and superprojective normed spaces in order to obtain duality relationships between strictly singular and strictly cosingular operators and their conjugates. We remark that all the above authors investigated only the case of bounded operators in Banach spaces. It is the purpose of this paper to consider this study in the more general setting of linear relations in arbitrary normed spaces.

In Section 2 we show that the definition of strictly cosingular linear relation generalises the classical definition of Pelczynski [15], with the two definitions being equivalent in the case of bounded operators from one Banach space into another. We present a result which will be used extensively in the subsequent sections.

Section 3 investigates when the conjugate of a strictly singular linear relation is strictly singular and, conversely, when the strict singularity of the conjugate implies the strict singularity of the linear relation.

In Section 4 we analyse the strictly cosingular linear relations in a similar way. The results obtained in Sections 3 and 4 generalise similar results of Whitley [17] and Shannon [16] for bounded operators between Banach spaces.

Notations. We recall some basic definitions from the theory of linear relations in normed spaces following the notation and terminology of the book [5]. Here $X$ and $Y$ are normed spaces. A linear relation or multivalued linear operator $T$ in $X \times Y$ is a mapping from a subspace $D(T) \subseteq X$, called the domain of $T$, into $P(Y) \backslash\{\theta\}$ such that

$$
T\left(\alpha x_{1}+\beta x_{2}\right)=\alpha T x_{1}+\beta T x_{2}
$$

Received 16th October, 2003

Supported in part by DGI (Spain), Proyecto BFM2001-1149.

Copyright Clearance Centre, Inc. Serial-fee code: 0004-9727/05 \$A2.00+0.00. 
for all nonzero scalars $\alpha, \beta \in K$ and $x_{1}, x_{2} \in D(T)$. The class of such relations $T$ is denoted by $L R(X, Y)$. If $T$ maps the points of its domain to singletons, then $T$ is said to be a single valued or simple operator.

The graph $G(T)$ of $T \in L R(X, Y)$ is

$$
G(T):=\{(x, y) \in X \times Y: x \in D(T), y \in T x\} .
$$

Let $\tilde{X}$ denote the completion of the normed space $X$. The completion $\tilde{T}$ of $T \in L R(X, Y)$ is the linear relation in $L R(\widetilde{X}, \widetilde{Y})$ whose graph is $\widetilde{G(T)}$.

Let $M$ be a subspace of $D(T)$. Then the restriction $\left.T\right|_{M}$ is defined by

$$
G\left(\left.T\right|_{M}\right):=\{(m, y): m \in M, y \in T m\} .
$$

For any subspace $M$ of $X$ such that $M \cap D(T) \neq \emptyset$, we write $\left.T\right|_{M}=\left.T\right|_{M \cap D(T)}$. The inverse of $T$ is the linear relation $T^{-1}$ defined by

$$
G\left(T^{-1}\right):=\{(y, x) \in Y \times X:(x, y) \in G(T)\} .
$$

If $T^{-1}$ is single valued, then $T$ is called injective, that is, $T$ is injective if and only if its null space $N(T):=T^{-1}(0)=\{0\}$, and $T$ is said to be surjective if its range $R(T):=T(D(T))=Y$. If $M$ is a subspace of $X$, then $M^{\perp}:=\left\{x^{\prime} \in X^{\prime}: x^{\prime}(x)\right.$ $=0$ for all $x \in M\}$. The conjugate $T^{\prime}$ of $T$ is defined by

$$
G\left(T^{\prime}\right):=G\left(-T^{-1}\right)^{\perp} \subseteq Y^{\prime} \times X^{\prime}
$$

where

$$
\left\langle(y, x),\left(y^{\prime}, x^{\prime}\right)\right\rangle:=\left\langle x, x^{\prime}\right\rangle+\left\langle y, y^{\prime}\right\rangle .
$$

For a given closed subspace $E$ of $X$ let $J_{E}^{X}$ (or simply, $J_{E}$ ) denote the injection map from $E$ into $X$ and $Q_{E}^{X}$ (or simply, $Q_{E}$ ) denote the quotient map from $X$ onto $X / E$. We shall denote $Q \frac{Y}{T(0)}$ by $Q_{T}$. Clearly $Q_{T} T$ is single valued. For

$$
x \in D(T),\|T x\|:=\left\|Q_{T} T x\right\|
$$

and the norm of $T$ is defined by $\|T\|:=\left\|Q_{T} T\right\|$. We shall write $J_{X}^{\tilde{X}}$ for the injection of $X$ into its completion.

The families of infinite dimensional and closed infinite codimensional subspaces of $X$ are denoted by $\mathcal{I}(X)$ and $\mathcal{E}(X)$ respectively. A linear relation $T \in L R(X, Y)$ is said to be continuous if for each neighbourhood $V$ in $R(T), T^{-1}(V)$ is a neighbourhood in $D(T)$, bounded if it is continuous and everywhere defined, open if its inverse is continuous, partially continuous if there exists a finite codimensional subspace $M$ of $X$ such that $\left.T\right|_{M}$ is continuous, semicontinuous if it is of the form $T=A+F$, where $A$ is continuous and $F$ has finite dimensional range, compact if $Q_{T} T$ is a compact single valued, $F_{+}$if 
there exists a finite codimensional subspace $M$ of $X$ for which $\left.T\right|_{M}$ is injective and open, $F_{-}$if $T^{\prime}$ is $F_{+}$. If $Y$ is infinite dimensional, then

$$
\begin{aligned}
\Gamma^{\prime}(T) & :=\inf \left\{\left\|Q_{M} J_{Y}^{\widetilde{Y}} T\right\|: M \in \mathcal{E}(\tilde{Y})\right\}, \\
\Delta^{\prime}(T) & :=\sup \left\{\Gamma^{\prime}\left(Q_{M} T\right): M \in \mathcal{E}(Y)\right\}
\end{aligned}
$$

and these quantities are defined to be zero if $Y$ is finite dimensional.

The class of partially continuous, $F_{+}$and $F_{-}$linear relations in $L R(X, Y)$ will be denoted by $P B(X, Y), F_{+}(X, Y)$ and $F_{-}(X, Y)$ respectively.

As remarked by Wilcox [18], single valued maps were favoured as the natural morphisms in the rigorous development of topology at the start of the 20th century. Nevertheless, limits of sequences of sets were considered by Painlevé in 1909 and later by Kuratowski [11] in 1958. Furthermore, extension to problems in topology led to the study of selections or single valued parts of upper and lower semicontinuous set valued maps. Multivalued maps, of course, occur quite naturally, but the earnest development of mathematical methods for set valued or multivalued problems came in the 1960's.

Linear relations were introduced by von Neumann [14], motivated by the need to consider conjugates of non densely defined linear differential operators.

Problems in optimisation and control also lead to the study of set valued maps and differential inclusions (see, for example, Aubin and Cellina [1], Clarke [2], among others). Studies of convex processes, tangent cones, etcetera, form part of the theory of convex analysis developed to deal with nonsmooth problems in viability and control theory, for example. Some of the basic topological properties from this area coincide with the core of the topological results for multivalued linear operators. Others works on multivalued linear operators include the treatise on partial differential relations by Gromov [9] and the application of multivalued methods to the solution of differential equations by Favini and Yagi [7].

A recent work on linear relations of semi-Fredholm type and others classes related to them is the book "Multivalued Linear Operators" by Cross [5]. This is the first book that has been published on these classes of linear relations.

Finally, according to Whitley [17], a normed space $X$ is called subprojective if for every closed infinite dimensional subspace $W$ of $X$, there exists a closed infinite dimensional subspace $W_{1}$ of $W$ such that $W_{1}$ is topological complemented in $X$. We say that $X$ is superprojective if for every $M \in \mathcal{E}(X)$ there exists $M_{1} \in \mathcal{E}(X)$ such that $M \subseteq M_{1}$ and $M_{1}$ is topological complemented in $X$.

\section{DEFinitions AND BASIC RESUltS}

We begin by giving some auxiliary properties of strictly singular and strictly cosingular linear relations which will be used extensively in the following sections. 
Definition 1: ([5, V. 1.1].) A linear relation $T \in L R(X, Y)$ is called strictly singular if there is no $M \in \mathcal{I}(D(T))$ such that $\left.T\right|_{M}$ is injective and open.

This notion was introduced for bounded operators in Banach spaces in Kato [10] and for unbounded operators in normed spaces in van Dulst [6] and Cross [4].

Definition 2: ([5, V. 5.18].) $T \in L R(X, Y)$ is said to be strictly cosingular if $\Delta^{\prime}(T)=0$.

According to Pelczynski [15] a bounded operator $T: X \rightarrow Y$, where $X$ and $Y$ are Banach spaces, is called strictly cosingular if there does not exist a closed infinite codimensional subspace $M$ of $Y$ for which $Q_{M} T$ is surjective. Following Labuschagne [12] an operator $T: D(T) \subseteq X \rightarrow Y \quad(X, Y$ normed spaces) is defined to be strictly cosingular if there is no $M \in \mathcal{E}(Y)$ such that $\left(Q_{M} T\right)^{\prime}$ has a continuous inverse. Note that from [8, II. 4.4] it follows that this notion is equivalent to Pelczynski's.

As is illustrated by the next Proposition, the definition given for strictly cosingular linear relations corresponds to that given by Labuschagne for strictly cosingular operators.

PROPOSITION 3. Let $T \in L R(X, Y)$ be single valued. Then $T$ is strictly cosingular if and only if there is no $M \in \mathcal{E}(Y)$ such that $\left(Q_{M} T\right)^{\prime}$ has a continuous inverse.

Proof: The result follows directly from the fact that $T$ is not strictly cosingular in the sense of Labuschagne if and only if there exists $M \in \mathcal{E}(Y)$ such that $Q_{M} T \in F_{-}[\mathbf{1 3}$, 1.2] equivalently $\Gamma^{\prime}\left(Q_{M} T\right)>0$.

The families of all strictly singular and strictly cosingular linear relations in $L R(X, Y)$ will be denoted by $S S(X, Y)$ and $S C(X, Y)$ respectively.

Proposition 4. ([4, 3] and [12, V. 1.2].) Let $T \in L R(X, Y)$ be single valued of the form $T=A+F$ where $A$ is a continuous operator and $F$ is a finite rank operator. Then:

(i) $T \in S S(S C)$ if and only if $A \in S S(S C)$.

(ii) $T^{\prime} \in S S(S C)$ if and only if $A^{\prime} \in S S(S C)$.

THEOREM 5. Let $T \in L R(X, Y)$. Then:

(i) If $T$ is partially continuous, then $T$ is strictly singular if $T^{\prime}$ is strictly cosingular.

(ii) If $D\left(T^{\prime}\right)$ is closed and finite codimensional in $Y^{\prime}$, then $T$ is strictly cosingular whenever $T^{\prime}$ is strictly singular.

Proof: (i) Let us consider the various cases for $T$ :

CASE 1. $T$ is a continuous single valued. In that case the assertion was proved by Labuschagne $[12$, V. 3.1].

CASE 2. $T$ is a partially continuous single valued. This is covered by Case 1 if we combine Proposition 4 with the fact that if $T \in P B(X, Y)$ and single valued, then $J_{Y}^{\tilde{Y}} T$ is semicontinuous [5, V. 9.2]. 
CASE 3. $T$ is a partially continuous linear relation. We consider the single valued $Q_{T} T$. Then we have that

$$
T \in P B \Leftrightarrow Q_{T} T \in P B, T \in S S \Leftrightarrow Q_{T} T \in S S
$$

and $T^{\prime} \in S C \Rightarrow\left(Q_{T} T\right)^{\prime} \in S C$ (as $\left(Q_{T} T\right)^{\prime}=T^{\prime} J_{T(0)^{\perp}}\left[5\right.$, III. 1.10] and $\Delta^{\prime}\left(T^{\prime} J_{T(0)^{\perp}}\right)$ $\leqslant\left\|J_{T(0) \perp}\right\| \Delta^{\prime}\left(T^{\prime}\right)$ [5, IV. 5.8]. Thus, substituting $Q_{T} T$ for $T$, the result follows from the Case 2.

(ii) For single valued the theorem is due to Labuschagne [12, V. 3.1].

Passing to the general case, we observe that $D\left(T^{\prime}\right)$ is closed and of finite codimension in $Y^{\prime}$ if and only if $T$ is partially continuous and $\operatorname{dim} T(0)<\infty[5, \mathrm{~V} .11 .3]$. Then $Q_{T} T$ is a partially continuous operator which is equivalent to that $D\left(\left(Q_{T} T\right)^{\prime}\right)$ is closed and finite codimensional in $\left(Q_{T} Y\right)^{\prime}[\mathbf{1 3}, 2.5],\left(Q_{T} T\right)^{\prime} \in S S$ whenever $T^{\prime} \in S S[\mathbf{5}, \mathrm{V} .2 .10]$ and as $\operatorname{dim} \overline{T(0)}<\infty$ we deduce from [5, IV. 5.6] that $T$ is strictly cosingular if and only if so is $Q_{T} T$. Consequently, the result follows from the corresponding result for single valued applied to $Q_{T} T$.

Any single valued $T$ for which $D\left(T^{\prime}\right)=\{0\}$ is not partially continuous by $[13,2.5]$ and hence $T \notin S S$ by $[4,6]$. Clearly $T^{\prime} \in S S \cap S C$ and $T \notin S C$ since $T^{\prime}$ is compact and has a continuous inverse. This remark shows that the property (i) (respectively, (ii)) of Theorem 5 may fail if $T$ is not assumed to be partially continuous (respectively, if $D\left(T^{\prime}\right)$ is not required closed and finite codimensional).

\section{STRICTLY SINGULAR LINEAR RELATIONS AND THEIR CONJUGATES}

In this section we investigate the influence of subprojectivity and superprojectivity on the implication $T \in S S \Rightarrow T^{\prime} \in S S$ and the converse, where $T$ is a linear relation from one normed space into another.

First we recall the following definition.

Definition 6: [5, IV 3.1] Given $T \in L R(X, Y)$ let $X_{T}$ denote the vector space $D(T)$ normed by $\|x\|_{T}:=\|x\|+\|T x\|, x \in D(T)$. Let $G_{T} \in L R\left(X_{T}, X\right)$ be the identity injection of $X_{T}$ into $X$ which is called the graph operator of $T$ and we write $G_{T}=G$ when $\mathrm{T}$ is understood.

Note that $T G$ is a bounded linear relation by [5, IV. 3.2].

Proposition 7. Let $Y$ be subprojective and $T \in S C(X, Y)$ such that $D\left(T^{\prime}\right)$ is closed and of finite codimension in $Y^{\prime}$. Then $T \in S S(X, Y)$.

Proof: According to [5, V. 11.3], $D\left(T^{\prime}\right)$ is closed and finite codimensional in $Y^{\prime}$ if and only if $T$ is partially continuous and $\operatorname{dim} T(0)<\infty$.

First assume that $T$ is bounded. Suppose that $T \notin S S(X, Y)$ and let $M \in I(X)$ for which $\left.T\right|_{M}$ is injective and open and so by [5, III. 4.6] $\left(T J_{M}\right)^{\prime}$ is open. Since 
$\operatorname{dim} D\left(\left.T\right|_{M}\right)+\left.\operatorname{dim} T\right|_{M}(0)=\operatorname{dim} D\left(\left(\left.T\right|_{M}\right)^{-1}\right)+\operatorname{dim}\left(\left.T\right|_{M}\right)^{-1}(0)[5$, I. 6.4], we have that the closure of $T M$, say $\overline{T M}$, is a closed infinite dimensional subspace of $Y$, and thus, by the subprojectivity of $Y$ there exists a topological complement subspace $N \in \mathcal{I}(\overline{T M})$ and let $Z \in \mathcal{E}(Y)$ be a topological complement of $N$.

To prove that $T$ is not strictly cosingular it is enough to show that $\Gamma^{\prime}\left(Q_{z} T\right)>0$ or equivalently $Q_{Z} T \in F_{-}$(as $\operatorname{dim} Q_{Z} T(0)<\infty$ and [5, V. 5.17]). Clearly $Q_{N^{\perp}} J_{Z^{\perp}}$ is an isomorphism and thus there exists $\alpha>0$ such that

$$
\alpha\left\|y^{\prime}\right\| \leqslant\left\|y^{\prime}+N^{\perp}\right\|, \quad y^{\prime} \in Z^{\perp} \cap D\left(T^{\prime}\right)
$$

We shall verify that $\left(T J_{M}\right)^{\prime}$ is an extension of $Q_{M^{\perp}} T^{\prime}$. From [5, III. 1.4] and $D(T)$ $=X$ (as $T$ is bounded) we obtain that $\left(T J_{M}\right)^{\prime}(0)=\left(Q_{M^{\perp}} T^{\prime}\right)(0)=\{0\}$ and by [5, III. 1.6], $G\left(Q_{M^{\perp}} T^{\prime}\right)=G\left(J_{M}^{\prime} T^{\prime}\right) \subseteq G\left(\left(T J_{M}\right)^{\prime}\right)$. Now, [5, I. 2.11] yields the desired property. From this fact combined with the openess of $\left(T J_{M}\right)^{\prime}$ it follows that there exists $\beta>0$ such that

In particular,

$$
\beta\left\|y^{\prime}+N\left(\left(T J_{M}\right)^{\prime}\right)\right\| \leqslant\left\|\left(T J_{M}\right)^{\prime} y^{\prime}\right\|, y^{\prime} \in D\left(\left(T J_{M}\right)^{\prime}\right) .
$$

$$
\beta\left\|y^{\prime}+N\left(\left(T J_{M}\right)^{\prime}\right)\right\| \leqslant\left\|Q_{M^{\perp}} T^{\prime} y^{\prime}\right\|, \quad y^{\prime} \in Z^{\perp} \cap D\left(T^{\prime}\right)
$$

We observe that $N\left(\left(T J_{M}\right)^{\prime}\right)=\overline{T M}^{\perp}\left(\left[5\right.\right.$, III. 1.4]) $\subseteq N^{\perp}$. Thus, as a consequence of (1) and (2), we get

$$
\alpha \beta\left\|y^{\prime}\right\| \leqslant \beta\left\|y^{\prime}+N^{\perp}\right\| \leqslant\left\|T^{\prime} y^{\prime}+M^{\perp}\right\| \leqslant\left\|T^{\prime} y^{\prime}\right\|, y^{\prime} \in Z^{\perp} \cap D\left(T^{\prime}\right) .
$$

Hence $T^{\prime} J_{Z^{\perp}} \in F_{+}$. But since $\left(Q_{Z} T\right)^{\prime}=T^{\prime} Q_{Z}^{\prime}=T^{\prime} J_{Z^{\perp}}$ [5, III. 1.6 and III. 1.9], we conclude that $\left(Q_{Z} T\right)^{\prime} \in F_{+}$equivalently $Q_{z} T \in F_{-}$, as required.

Turning to the general case, if $D\left(T^{\prime}\right)$ is closed and finite codimensional in $Y^{\prime}$ then so is $T G$ [5, V. 11.3]). Also $T \in S S \Leftrightarrow T G \in S S$ by [5, IV. 3.4] and by [5, IV. 5.8], $T G \in S C$ if $T \in S C$. Therefore, the result follows applying the bounded case to $T G$.

THEOREM 8. Let $Y$ be subprojective and $T^{\prime} \in S S\left(Y^{\prime}, X^{\prime}\right)$ such that $D\left(T^{\prime}\right)$ is closed and finite codimensional in $Y^{\prime}$. Then $T \in S S(X, Y)$.

Proof: Combine Theorem 5 (ii) with Proposition 7.

This Theorem generalises the analogous result for bounded operators in Banach spaces of Whitley $[\mathbf{1 7}]$

PROPOSITION 9. Let $\widetilde{D(T)}$ be superprojective and let $T \in S S(X, Y)$ such that $D\left(T^{\prime}\right)$ is closed and finite codimensional in $Y^{\prime}$. Then $T \in S C(X, Y)$.

Proof: Proceeding as in the proof of Theorem 5, we may suppose (substituting $Q_{T} T$ for $T$ if necessary) that $T$ is single valued. Whithout loss of generality let $D(T)=X$. Since

$$
T^{\prime} \equiv\left(J_{Y}^{\tilde{Y}} T\right)^{\prime}, T \in S S \Leftrightarrow J_{Y}^{\tilde{Y}} T \in S S
$$


and $T$ is strictly cosingular if so is $J_{Y}^{\tilde{Y}} T$ (as $\Delta^{\prime}(T) \leqslant \Delta^{\prime}\left(J_{Y}^{\tilde{Y}} T\right)$ by [5, IV. 5.12]), we may suppose that $Y$ is complete. Now, $T$ is partially continuous (as $T$ is strictly singular) and thus $T$ is semicontinuous by [5, V. 9.2]. Hence by Proposition 4 we may assume $T$ to be continuous and since $Y$ is Banach, there exists an unique continuous extension of $T$ to all of $\tilde{X}$, say $\bar{T}$, such that $T \in S S(S C) \Leftrightarrow \bar{T} \in S S(S C)$ by $[3,1.11]$ and $[4,2.7]$. In consequence it is enough to consider the case when $T \in L R(X, Y)$ is a bounded strictly singular operator, $X$ and $Y$ are complete and $X$ is superprojective. But, in this case the result was proved by Whitley [17].

Proposition 10. [5, IV. 3.17] Let $T \in L R(X, Y)$. There exists a normed space $Z$ and a bounded operator $H_{T}$ mapping $Y$ onto $Z$ with the following properties:

(i) $H_{T} T$ is a continuous single valued.

(ii) $Z^{\prime}=D_{T^{\prime}}, H_{T}^{\prime}=G_{T^{\prime}}$ and hence $\left(H_{T} T\right)^{\prime}=T^{\prime} G_{T^{\prime}}$.

THEOREM 11. Let $T \in L R(X, Y)$ such that $D\left(T^{\prime}\right)$ is a superprojective Banach space with $\operatorname{dim} Y^{\prime} / D\left(T^{\prime}\right)<\infty$ and $T^{\prime} \in S S\left(Y^{\prime}, X^{\prime}\right)$. Then $T \in S S(X, Y)$.

Proof: First assume that $T$ is single valued. In that case $T^{\prime}$ is continuous and single valued (as $D\left(T^{\prime}\right)$ is closed and finite codimensional $\Leftrightarrow T$ is partially continuous by $[13,2.5]$ and the conjugate of a partially continuous linear relation is continuous by [5, V. 9.6]). The result follows as an immediate consequence of Proposition 9 applied to $T^{\prime}$ and Theorem 5 (i).

Now let $T$ be an arbitrary linear relation. Since $R\left(H_{T}^{\prime}\right)=D_{T^{\prime}}=D\left(\left(H_{T} T\right)^{\prime}\right)$ is isomorphic to $D\left(T^{\prime}\right)$ under the operator $H_{T^{\prime}}$ (Proposition 10), we have that $D\left(\left(H_{T} T\right)^{\prime}\right)$ is a superprojective Banach space of finite codimension and $H_{T}^{\prime} \in F_{-}$equivalently $H_{T} \in F_{+}$. Furthermore, $T^{\prime} \in S S$ if and only if $T^{\prime} G_{T^{\prime}}=\left(H_{T} T\right)^{\prime} \in S S$ by [5, IV. 3.4] and hence from what has been proved for the single valued case, $H_{T} T$ is strictly singular. Suppose that $T$ is not strictly singular and let $M \in \mathcal{I}(D(T))$ for which $\left.T\right|_{M} \in F_{+}$. Then, since $H_{T} \in F_{+}$ we deduce from $[18,5.4 .1]$ that $\left.H_{T} T\right|_{M} \in F_{+}$and so $H_{T} T$ is not strictly singular.

For bounded operators in Banach spaces this Theorem was obtained by Whitley [17].

THEOREM 12. Let $T \in S S(X, Y)$ such that $D(T)$ is reflexive and $D\left(T^{\prime}\right)$ is superprojective. Then $T^{\prime} \in S S\left(Y^{\prime}, X^{\prime}\right)$

Proof: Since $H_{T} T$ is strictly singular if so is $T, T^{\prime}$ is strictly singular if and only if $\left(H_{T} T\right)^{\prime}$ is strictly singular and since $T^{\prime} \equiv\left(J_{Y}^{\bar{Y}} T\right)^{\prime}$ with $T \in S S$ if and only if $J_{Y}^{\bar{Y}} T \in S S$, we may thus suppose without loss of generality that $T$ is continuous single valued and $Y$ is complete. The proof now reduces to the standard one for bounded operators in Banach spaces of Whitley [17]. 


\section{STRICTLY COSINGULAR LINEAR RELATIONS AND THEIR CONJUGATES}

In this section we study the strictly cosingular linear relations, paralleling our investigation of strictly singular linear relations of the previous section.

Theorem 13. Let $T \in L R(X, Y)$ such that $D(T)^{\prime}$ is subprojective, $D\left(T^{\prime}\right)$ is closed and of finite codimension in $Y^{\prime}$ and $T^{\prime}$ is strictly cosingular. Then $T$ is strictly cosingular.

Proof: Proceeding as in the proof of Theorem 5, we may assume (substituting $Q_{T} T$ for $T$ if necessary) that $T$ is single valued. In that case, the result follows directly from Theorem 5 (ii) and Proposition 7 upon observing the fact that $T^{\prime}$ is continuous and single valued (as $T$ is partially continuous) if and only if $T^{\prime \prime}$ is bounded [5, VIII. 1.5]. $]$

For bounded operators in Banach spaces, the corresponding result was proved by Shannon [16].

THEOREM 14. Let $T \in L R(X, Y)$ such that $\widetilde{D(T)}$ is a superprojective space, $T^{\prime} \in S C\left(Y^{\prime}, X^{\prime}\right)$ with $D\left(T^{\prime}\right)$ a closed subspace of $Y^{\prime}$ and $\operatorname{dim} Y^{\prime} / D\left(T^{\prime}\right)<\infty$. Then $T \in S C(X, Y)$.

ProOF: Consider Theorem 5 (i) alongside Proposition 9.

This Theorem is a generalisation of Shannon [16] in the sense that $T$ is not required to be a continuous operator.

Theorem 15. Let $T \in L R(X, Y)$ with $X$ and $Y$ reflexive and $D\left(T^{\prime}\right)$ is a superprojective space, closed and finite codimensional in $Y^{\prime}$. Then $T^{\prime}$ is strictly cosingular if $\widetilde{T}$ is strictly cosingular and everywhere defined.

Proof: Since $D\left(T^{\prime}\right)$ is closed and finite codimensional in $Y^{\prime}$ and $D(\widetilde{T})=X$ we have that $\tilde{T}$ is bounded and $\operatorname{dim} \tilde{T}(0)<\infty$ (by [5, V. 11.3]), $\widetilde{T} \in S C \Leftrightarrow Q_{\tilde{T}} \widetilde{T} \in S C$ (by [5, IV. 5.6]), $T^{\prime}$ is a continuous single valued with $\left(Q_{\tilde{T}} \tilde{T}\right)^{\prime \prime}=T^{\prime \prime}$ (by [5, VIII. 1.3 and VIII. 1.5]). Furthermore, as $X$ and $Y / \widetilde{T}(0)$ are reflexive, it is clear that the operator $Q_{\tilde{T}} \tilde{T}$ is strictly cosingular if and only if so is $\left(Q_{\tilde{T}} \tilde{T}\right)^{\prime \prime}$. The result now follows from Theorem 14.

\section{REFERENCES}

[1] J.P. Aubin and A. Cellina, Differential inclusions. Set valued maps and viability theory (Springer-Verlag, Berlin, New York, 1984).

[2] F.H. Clarke, Optimization and nonsmooth analysis, Canadian Mathematical Society Series of Monographs and Advanced Texts (J. Wiley and Sons, Toronto, 1983).

[3] R.W. Cross, 'Properties of some norm related functions of unbounded linear operators', Math. Z. 199 (1988), 285-303.

[4] R.W. Cross, 'Unbounded strictly singular operators', Nederl. Akad. Wetensch. Indag. Math. 91 (1988), 245-248. 
[5] R.W. Cross, Multivalued linear operators, Monographs and Textbooks in Pure and Applied Mathematics 213 (Marcel Dekker, New York, 1998).

[6] D. van Dulst, 'On strictly singular operators', Compositio Math 23 (1971), 169-183.

[7] A. Favini and A. Yagi, 'Multivalued linear operators and degenerate evolution equations', Ann. Mat. Pura. Appl. (4) 163 (1993), 353-384.

[8] S. Goldberg, Unbounded linear operators. Theory and Applications (McGraw-Hill, New York, 1966).

[9] M. Gromov, Partial differential relations (Springer-Verlag, Berlin, 1986).

[10] T. Kato, 'Perturbation theory for nullity, deficiency, and other quantities of linear operators', J. Anal. Math. 6 (1958), 273-322.

[11] C. Kuratowski, Topology I (Polska Akademia Nauk, Varsovia, 1952).

[12] L.E. Labuschagne, Functions of operators and the classes associated with them, (Ph. D. thesis) (Univ. Cape Town, 1988).

[13] L.E. Labuschagne, 'Characterisations of partially continuous, strictly cosingular and $\phi_{-}$-type operators', Glasgow Math. J. 33 (1991), 203-212.

[14] J. von Neumann, Functional Operators, II. The Geometry of Orthogonal spaces, Annals of Math. Studies 22 (Princeton University Press, Princeton, N.J., 1950).

[15] A. Pelczynski, 'On strictly singular and strictly cosingular operators I, II', Bull. Acad. Polon. Sci. Ser. Math. Astr. Phys 13 (1965), 31-41.

[16] G.P. Shannon, 'Strictly singular and strictly cosingular operators on topological vector spaces', Proc. Royal Irish Acad. Sci. Sect. A 73 (1973), 303-308.

[17] R.J. Whitley, 'Strictly singular operators and their conjugates', Trans. Amer. Math. Soc 113 (1964), 252-261.

[18] D. Wilcox, Multivalued semi-Fredholm operators in normed linear spaces, (Ph.D. Thesis) (Univ. Cape Town, 2001).

Department of Mathematics

University of Oviedo,

33007, Oviedo

Spain

e-mail: seco@pinon.ccu.uniovi.es 